\title{
Every knot is a billiard knot
}

\author{
P. -V. Koseleff \& D. Pecker
}

July 10, 2017

\begin{abstract}
We show that every knot can be realized as a billiard trajectory in a convex prism. This solves a conjecture of Jones and Przytycki.
\end{abstract}

keywords: Billiard knots, Lissajous knots, Chebyshev knots, Cylinder knots

Mathematics Subject Classification 2000: 14H50, 57M25, 14P99

\section{Introduction}

The study of billiard trajectories in a polyhedron was introduced in 1913 by König and Szücs in $[\mathrm{KS}]$. They proved density results for a billiard trajectory in a cube. Their theorem is strongly related to the famous Kronecker density theorem (see $[\mathrm{HW}]$ ).

More recently, Jones and Przytycki considered the periodic billiard trajectories with no self-intersection as knots. They proved that billiard knots in a cube are isotopic to Lissajous knots, and deduced that not all knots are billiard knots in a cube ([JP], see also [La, C, BHJS, BDHZ]). They also proved that every torus knot (or link) of type $(n, k)$, where $n \geq 2 k+1$ can be realized as a billiard knot in a cylinder (or in a prism with a regular $n$-gonal floor). Przytycki went deeper into the study of symmetrical billiards in $[\mathrm{P}]$.

Lamm and Obermeyer [LO] proved that billiard knots in a cylinder are either periodic or ribbon, hence not all knots are billiard knots in a cylinder. In $[\mathrm{KP}]$ we constructed many other examples of billiard knots in convex polyhedrons (in fact irregular truncated cubes). Dehornoy constructed in [D] a billiard which contains all knots, but this billiard is not convex.

In this paper we prove the following conjecture of Jones and Przytycki:

Every knot is a billiard knot in some convex polyhedron.

Our result is more precise:

Theorem 8 Every knot (or link) is a billiard knot (or link) in some convex right prism.

Using a theorem of Manturov [M], we first prove that every knot has a diagram which is a star polygon. Then, perturbing this polygon, we obtain an irregular diagram of the same knot. We deduce that it is possible to suppose that 1 and the arc lengths of the crossing points are linearly independent over $\mathbf{Q}$. Then, it is possible to use the classical Kronecker density theorem to prove our result. 


\section{Every knot has a projection which is a star polygon}

A toric braid is a braid corresponding to the closed braid obtained by projecting the standardly embedded torus knot into the $x y$-plane. A toric braid is a braid of the form $\tau_{k, n}=\left(\sigma_{1} \sigma_{2} \cdots \sigma_{k-1}\right)^{n}$, where $\sigma_{1}, \ldots, \sigma_{k-1}$ are the standard generators of the full braid group $B_{k}$. A quasitoric braid of type $(k, n)$ is a braid obtained by changing some crossings in the toric braid $\tau_{k, n}$. The quasitoric braids form a subgroup of $B_{k}$. Consequently there exist trivial quasitoric braids of arbitrarily great length, and any quasitoric braid is equivalent to a quasitoric braid of type $(k, n)$ with $n \geq 2 k+1$.

Manturov's theorem tells us that every knot (or link) is realized as the closure of a quasitoric braid $([\mathrm{M}])$.

The following definition of polygonal stars will be useful for links.

Definition 1 Let $p, q$ be integers. The polygonal star $\left\{\frac{p}{q}\right\} \subset \mathbf{R}^{2}$ is given by its vertices $e(k)=e^{\frac{2 k i \pi}{p}}$, and its sides $(e(k), e(k+q)), k=0, \ldots, p-1$.

When $p$ and $q$ are coprime integers, this is the usual definition of star polygons. The following picture shows the polygonal stars $\left\{\frac{10}{3}\right\},\left\{\frac{10}{2}\right\}$ and $\left\{\frac{9}{3}\right\}$, as projections of billiard torus links $T(10,3), T(10,2)$ and $T(9,3)$. The dotted lines correspond to the parts $z<0$ of the link.
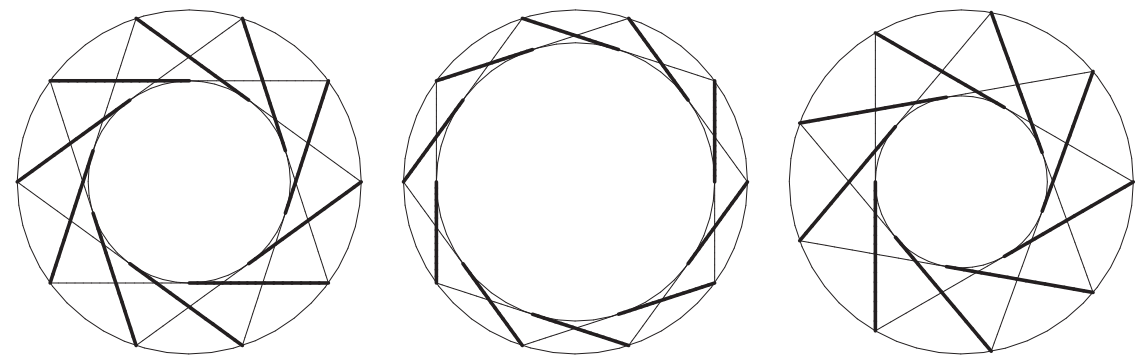

Figure 1: The polygonal stars $\left\{\frac{10}{3}\right\},\left\{\frac{10}{2}\right\}$ and $\left\{\frac{9}{3}\right\}$, projections of the torus links $T(10,3)$, $T(10,2)$ and $T(9,3)$.

Theorem 2 Every knot (or link) has a projection that is a polygonal star.

Proof. Let our knot be realized as the closure of a quasitoric braid of type $(k, n)$. By our remark, we can suppose $n \geq 2 k+1$. Now, we use the result of Jones and Przytycki which says that every torus knot of type $(k, n), n \geq 2 k+1$ can be realized as a billiard knot in a cylinder. In their construction, the projection on the $x y$-plane is the closure of the toric braid $\tau_{k, n}$, which proves our result. 
Remark 3 It is also possible to use a theorem of Lamm and Obermeyer [LO] to give another proof of theorem 1 in the knot case. A Rosette braid is a braid of the form $\left(\sigma_{1}^{\varepsilon_{1}} \cdots \sigma_{k-1}^{\varepsilon_{k-1}}\right)^{n}, \varepsilon_{i}= \pm 1$, and a Rosette knot is the closure of a Rosette braid. The theorem of Lamm and Obermeyer tells us that every Rosette knot can be represented by a billiard knot in a cylinder. The knot diagrams obtained in their proof are star polygons isotopic to the closures of some quasitoric braids.

\section{$3 \quad$ Breaking the symmetry}

Since we want to obtain all knots, we need irregular diagrams.

First, let us recall some facts about billiard trajectories. If $A B C$ is a piece of a polygonal line, then the mirror placed at $B$, is the hyperplane $\mu(B)$ which is orthogonal to the internal bisector of $\widehat{B}$ at $B$. The mirror room at $B$ is the closed half-space containing $A, B, C$ and the mirror at $B$.

We define a billiard trajectory to be a finite union of polygonal lines, which is contained in all its mirror rooms. A billiard knot (or link) is a polygonal knot (or link) ([A, C]) which is a billiard trajectory.

The following result allows us to forget about the billiard, and focus our attention on the trajectory. It is valid in every dimension.

Lemma 4 Let $\mathcal{Q}=\left(Q_{0}, \ldots, Q_{n-1}\right)$, be a billiard trajectory such that $\mathcal{Q} \cap \mu\left(Q_{k}\right)=Q_{k}$. Then, if $\mathcal{P}=\left(P_{0}, \ldots, P_{n-1}\right)$ is sufficiently close to $\mathcal{Q}$, it is a billiard trajectory in some convex polyhedron.

Proof. Let $\overrightarrow{u_{k}}(\mathcal{Q})$ be the unit vector of the internal bisector of $\widehat{Q_{k}}$. The hypothesis means that for every $k, i, i \neq k$, the scalar product $\overrightarrow{u_{k}}(\mathcal{Q}) \cdot \overrightarrow{Q_{k} Q_{i}}$ is positive.

Since $\overrightarrow{u_{k}}(\mathcal{Q})$ and $\overrightarrow{Q_{k} Q_{i}}$ depend continuously on $\mathcal{Q}$, this condition remains true for any trajectory $\mathcal{P}$ that is sufficiently close to $\mathcal{Q}$.

Proposition 5 Let $K$ be a knot. There exists a plane billiard trajectory $\mathcal{P}$ which is a projection of a knot isotopic to $K$, and which satisfies the following irregularity condition:

If $t_{i}$ are the arc lengths corresponding to the crossings of $\mathcal{P}$, then the numbers $1, t_{i}$ are linearly independent over $\mathbf{Q}$.

Proof. Let $\mathcal{Q}=\left(Q_{0}, \ldots, Q_{n-1}\right), Q_{n}=Q_{0}$ be a star polygon which is a projection of $K$. Let us suppose that each line $\left(Q_{k} Q_{k+1}\right)$ has an equation of the form $y=\alpha_{k} x+\beta_{k}$. Then, if $\left(a_{k}, b_{k}\right)$ are sufficiently close to $\left(\alpha_{k}, \beta_{k}\right)$, the lines $\left\{y=a_{k} x+b_{k}\right\}$ determine a nonconvex polygon $\mathcal{P}=\left(P_{0}, \ldots, P_{n-1}\right)$ close to $\mathcal{Q}$. By our lemma, $\mathcal{P}$ is a periodic billiard trajectory in some convex polygon.

By Baire's theorem, we can suppose that the numbers $a_{0}, a_{1}, \ldots, a_{n-1}$ and $b_{0}, b_{1}, \ldots, b_{n-1}$ are algebraically independent over $\mathbf{Q}$. 


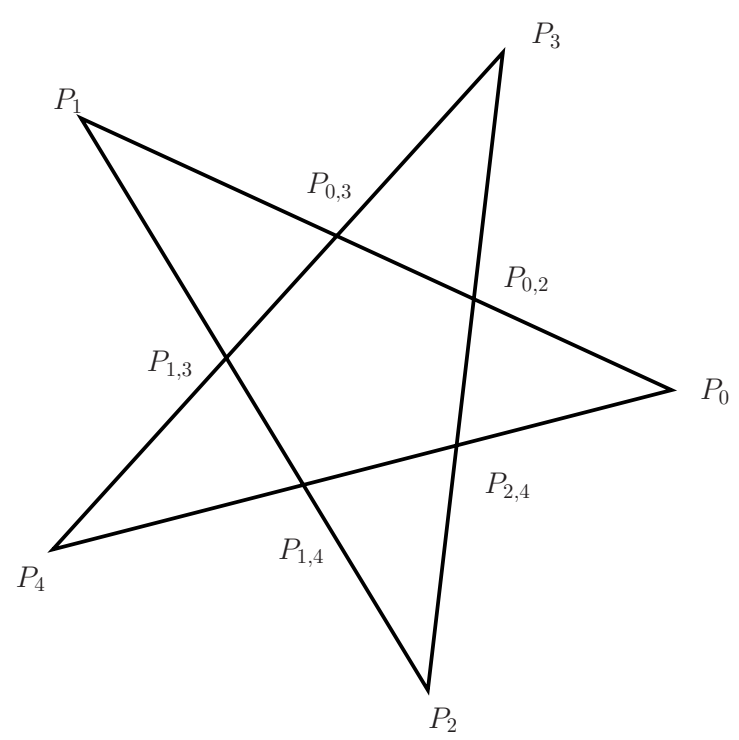

Figure 2: Naming the vertices and crossings of a pentagonal trajectory.

Let $I$ be the set of integer pairs $(i, j), j \neq i-1$, such that the intersection of $\left[P_{i}, P_{i+1}\right]$ and $\left[P_{j}, P_{j+1}\right]$ is a point $P_{i, j}$.

The vertex $P_{i}$ is the point $P_{i-1, i}$. The abcissa of $P_{i, j}$ is $x_{i, j}=\frac{b_{i}-b_{j}}{a_{j}-a_{i}}$, and the length of $\left[P_{i}, P_{i, j}\right]$ is $\left|\ell_{i, j}\right|$, where $\ell_{i, j}=\sqrt{1+a_{i}^{2}}\left(x_{i, j}-x_{i-1, i}\right)$.

Let us show that the numbers $\ell_{i, j}$ and 1 are linearly independent over $\mathbf{Q}$. Suppose that we have a linear relation with rational coefficients $\sum_{(i, j) \in I} \lambda_{i, j} \ell_{i, j}=\lambda$, with $\lambda, \lambda_{i, j} \in \mathbf{Q}$.

This is an algebraic relation between the $a_{i}$ and the $b_{i}$. Since these numbers are algebraically independent over $\mathbf{Q}$, this relation must be an identity.

Let $k \leq n-1$ be a fixed non-negative integer, and let us substitute $b_{k}=1$ and $b_{i}=0$ if $i \neq k$ in this identity.

We obtain a new identity between the $a_{i}$.

$$
\begin{array}{r}
\sum_{j=0}^{n-1} \lambda_{k, j} \sqrt{1+a_{k}^{2}}\left(\frac{1}{a_{j}-a_{k}}-\frac{1}{a_{k-1}-a_{k}}\right)-\left(\sum_{j=0}^{n-1} \lambda_{k+1, j}\right) \frac{\sqrt{1+a_{k+1}^{2}}}{a_{k+1}-a_{k}} \\
+\sum_{i=0}^{n-1} \lambda_{i, k} \frac{\sqrt{1+a_{i}^{2}}}{a_{i}-a_{k}}=\lambda
\end{array}
$$

where $\lambda_{k, k}=\lambda_{k, k-1}=\lambda_{k+1, k}=0$. Substituting $a_{k}=\sqrt{-1}$, in this identity, we obtain

$$
-\left(\sum_{j=0}^{n-1} \lambda_{k+1, j}\right) \frac{\sqrt{1+a_{k+1}^{2}}}{a_{k+1}-\sqrt{-1}}+\sum_{i=0}^{n-1} \lambda_{i, k} \frac{\sqrt{1+a_{i}^{2}}}{a_{i}-\sqrt{-1}}=\lambda .
$$


Let $h \neq k+1$ be an integer, and let $a_{h} \rightarrow \sqrt{-1}$. From $\lim _{z \rightarrow \sqrt{-1}} \frac{\sqrt{1+z^{2}}}{z-\sqrt{-1}}=\infty$, we obtain $\lambda_{h, k}=0$. Since this is true for every $h$ and $k$, we deduce that $\lambda_{i, j}=0$ for all $(i, j) \in I$.

Finally, since the arc lengths of the points $P_{i, j}$ are given by $t_{i, j}=\left|\ell_{0,1}\right|+\left|\ell_{1,2}\right|+\cdots+$ $\left|\ell_{i-1, i}\right|+\left|\ell_{i, j}\right|$, we deduce the result.

Proposition 6 Let $L$ be a link. There exists a plane billiard trajectory $\mathcal{P}$ which is a projection of a link isotopic to $L$, and which satisfies the following condition.

If $\mathcal{R}$ is a component of $\mathcal{P}$ parametrized by arc length, and if $t_{i}$ are the arc lengths corresponding to the crossings, then the numbers $1, t_{i}$ are linearly independent over $\mathbf{Q}$.

Proof. The proof is almost identical to the preceding one.

There is a link isotopic to $L$ whose plane projection is a union of polygons

$$
\mathcal{P}=\mathcal{P}^{(1)} \cup \mathcal{P}^{(2)} \cup \cdots \cup \mathcal{P}^{(d)}
$$

whose vertices are $\left(P_{0}, P_{1}, \ldots, P_{N-1}\right)$. Let $\mathcal{R}$ be a component of $\mathcal{P}$, we can suppose that the vertices of $\mathcal{R}$ are $\left(P_{0}, P_{1}, \ldots, P_{n-1}\right)$.

Furthermore, we can suppose that the equations $y=a_{k} x+b_{k}$ of the sides of $\mathcal{P}$ are such that the numbers $a_{k}$ and $b_{k}, k=1, \ldots, N-1$, are algebraically independent over $\mathbf{Q}$.

Here, we consider the set $I$ of integer pairs $(i, j), i \in\{0, \ldots, n-1\}, j \in\{0, \ldots, N-$ $1\}, j \neq i-1$, corresponding bijectively to the arc lengths of the vertices and crossings contained in $\mathcal{R}$.

Then, the rest of the proof is exactly the same as in the case of knots.

\section{Proof of the theorem}

We will use Kronecker's theorem ([HW, Theorem 443]):

Theorem 7 (Kronecker (1884)) If $\theta_{1}, \theta_{2}, \ldots, \theta_{k}, 1$ are linearly independent over $\mathbf{Q}$, then the set of points $\left(\left(n \theta_{1}\right), \ldots,\left(n \theta_{k}\right)\right)$ is dense in the unit cube. Here $(x)$ denotes the fractional part of $x$.

Now, we can prove our main theorem.

Theorem 8 Every knot (or link) is a billiard knot (or link) in some convex prism.

Proof. First, we consider knots. By Theorem 2 there exists a knot isotopic to $K$ whose projection on the $x y$-plane is a periodic billiard trajectory in a convex polygon $\mathbf{D}$. If $t_{i}$ are the arc lengths corresponding to the crossings, we can suppose by Proposition 5 that the numbers $t_{1}, \ldots, t_{k}, 1$ are linearly independent over $\mathbf{Q}$. Using a dilatation, we can suppose that the total length of the trajectory is 1 . 
Consider the polygonal curve defined by $(x(t), y(t), z(t))$, where $z(t)$ is the sawtooth function $z(t)=2|(n t+\varphi)-1 / 2|$ depending on the integer $n$ and on the real number $\varphi$. If the heights $z\left(P_{k}\right)$ of the vertices are such that $z\left(P_{k}\right) \neq 0,1$, then it is a periodic billiard trajectory in the prism $\mathbf{D} \times[0,1]$ (see [JP, La, LO, P, KP]). If we set $\varphi=1 / 2+z_{0} / 2$, $\left.z_{0} \in\right] 0,1\left[\right.$, we have $z(0)=z_{0}$. Now, using Kronecker's theorem, there exists an integer $n$ such that the numbers $z\left(t_{i}\right)$ are arbitrarily close to any chosen collection of heights, which implies the result.

The case of links is similar. First, we find a plane billiard diagram of our link, and then we parameterize each component.

Remark 9 If the diagram has some regularity, then it is generally impossible to use Kronecker's theorem. This is illustrated by Lissajous knots and cylinder knots. This is also true for more general diagrams.

For example, suppose that there are four crossings of arc lengths $t_{1}, t_{2}, t_{3}, t_{4}$ such that $t_{2}-t_{1}=t_{4}-t_{3}$. So, if $z(t)=2\left|(n t+\varphi)-\frac{1}{2}\right|$ is the height function, then $\varepsilon_{i} z_{i}=2\left(n t_{i}+\varphi\right)-1$, with $\varepsilon_{i}= \pm 1$. We deduce that $\varepsilon_{1} z_{1}-\varepsilon_{2} z_{2}-\varepsilon_{3} z_{3}+\varepsilon_{4} z_{4} \in 2 \mathbf{Z}$. Consequently, we see that $z_{1}=z_{2}=z_{3}=1$ implies $z_{4}=1$. This clearly shows that the heights of the crossings cannot be chosen arbitrarily.

\section{References}

[A] C. Adams, The Knot Book: an Elementary Introduction to the Mahematical Theory of Knots. American Mathematical Society, Providence. 2004.

[BHJS] M. G. V. Bogle, J. E. Hearst, V. F. R. Jones, L. Stoilov, Lissajous knots, Journal of Knot Theory and its Ramifications, 3(2), pp. 121-140, 1994.

[BDHZ] A. Boocher, J. Daigle, J. Hoste, W. Zheng, Sampling Lissajous and Fourier knots, $\backslash$ protect \vrule width0pt \protect \href\{http://arxiv.org/abs/0707.4210\}\{arXiv:0707.4210\} 2007.

[C] P. Cromwell, Knots and links , Cambridge University press, Cambridge, 2004.

[D] Pierre Dehornoy, A billiard containing all links Preprint 2011.

[HW] G.H. Hardy, E.M. Wright, An Introduction to the Theory of Numbers, 4th edition, Oxford University Press, 1960.

[JP] V. F. R. Jones, J. Przytycki, Lissajous knots and billiard knots, Banach Center Publications, 42, pp. 145-163, 1998.

[KP] P. -V. Koseleff, D. Pecker, Chebyshev knots, Journal of Knot Theory and Its Ramifications, Vol 20, 4 (2011) 1-19 
[KS] D. König, A. Szücs, Mouvement d'un point abandonné à l'intérieur d'un cube, Rend. Circ. Mat. Palermo, Vol. 36 (1913), 79-90

[La] C. Lamm, There are infinitely many Lissajous knots, Manuscripta Math., 93, pp. 2937, 1997.

[LO] C. Lamm, D. Obermeyer, Billiard knots in a cylinder, J. Knot Theory Ramifications 8 (1999), no. 3, 353-366.

[M] V. O. Manturov, A combinatorial representation of links by quasitoric braids, European J. Combin. 23 (2002), no 2, 207-212.

[P] J. Przytycki, Symmetric knots and billiard knots, Chapter 20 of the book Ideal Knots, ed. A. Stasiak, V. Katrich and L. Kauffman, Series on Knots and Everything, Vol 19, World Sientific, River Edge, NJ, (1999) 374-414.

P. -V. Koseleff, UPMC-Paris 6, IMJ and INRIA Paris-Rocquencourt, koseleff@math. jussieu .fr D. Pecker, UPMC-Paris 6, Mathematics, pecker@math.jussieu.fr 